\title{
Expression and regulation of IL-22 in the IL-17-producing CD4+ T lymphocytes
}

\author{
Yeonseok Chung ${ }^{1}$, Xuexian Yang ${ }^{1}$, Seon Hee Chang ${ }^{1}$, Li Ma², Qiang Tian², Chen Dong ${ }^{1}$ \\ ${ }^{1}$ Department of Immunology, MD Anderson Cancer Center, Houston, TX 77030, USA; ${ }^{2}$ Institute for Systems Biology, Seattle, WA \\ 98103, USA
}

IL-22 is a novel cytokine in the IL-10 family that functions to promote innate immunity of tissues against infection. Although CD4+ helper T lymphocytes (TH) were found as a source of IL-22, the regulation of this cytokine has been poorly understood. Here, we show that IL-22 is expressed at both mRNA and protein levels by a novel subset of TH cells that also makes IL-17. IL-22 and IL-17 were found to be coordinately regulated by TGF $\beta$ and IL- 6 during TH differentiation by real-time PCR as well as ELISA analysis. However, IL-22 does not regulate TH differentiation; exogenous IL-22 or an IL-22 antagonist had no effect on TH differentiation. These data demonstrate a novel cytokine expressed by IL-17-producing T cells, and suggest interaction and synergy of IL-22 and IL-17 signaling pathways in tissue inflammation and autoimmune diseases.

Cell Research (2006) 16:902-907. doi:10.1038/sj.cr.7310106; published online 7 November 2006

Keywords: cytokines, helper T cells, $\mathrm{T}$ cell activation, gene regulation

\section{Introduction}

Following activation by antigen-presenting cells, CD4+ helper $\mathrm{T}$ lymphocytes $(\mathrm{TH})$ differentiate into effector $\mathrm{TH}$ cells that are specialized in their cytokine secretion and immunoregulatory function. TH cells have been historically classified into TH1 and TH2 cells $[1,2]$. TH1 cells make IFN $\gamma$ and regulate antigen presentation and cellular immunity against intracellular pathogens. TH2 cells produce IL$4,-5$ and -13 to regulate humoral responses and anti-parasite immunity. TH1 and $\mathrm{TH} 2$ differentiation is regulated by cytokine environment. IL-12 regulates TH1 differentiation while IL-4 is essential in TH2 differentiation.

Recently, a third subset of TH cells, named as THIL-17, TH-17 or inflammatory TH (THi), that produce IL-17 was identified to mediate pathogenic inflammatory response [3]. IL-17 regulates chemokines and other proinflammatory gene expression in vitro and in vivo [4]. IL-17 expression in TH cells was initially found to be regulated by ICOS costimulatory receptor [5]. Subsequently, IL-23, a cytokine

Correspondence: Chen Dong

Tel: +1-713-563-3203; Fax: +1-713-563-0604;

E-mail: cdong@mdanderson.org

Received 26 September 2006; revised 6 October 2006; accepted 10 October 2006; published online 7 November 2006 in the IL-12 family, was found to regulate IL-17 expression and expansion of IL-17-expressing T cells [6]. Importantly, impaired IL-17 expression caused by ICOS- or IL-23-deficiency was reported to result in resistance to autoimmune diseases. More recently, IL-6 in the presence of TGF $\beta$ has been shown by multiple investigators to initiate the differentiation of IL-17-expressing T cells in the absence of IL-23 [7], further supporting a distinct cytokine requirement in generation of IL-17-expressing TH cells.

IL-22 is a recently identified cytokine that belong to the IL-10 family $[8,9]$. In contrast to the anti-inflammatory function by IL-10, IL-22 does not inhibit proinflammatory cytokine expression by the innate immune system [8]. Instead, it was found to regulate the expression of $\beta$-defensin genes in keratinocytes possibly through activation of STAT3 [10]. Thus, IL-22 may regulate innate immunity and inflammation in the skin and its overexpression has been associated with dermatitis and psoriasis [11].

IL-22 expression was first discovered to be expressed by CD4+ T cells [8]. However, it is unknown what subset of TH cells express IL-22, or how it is regulated. Here, we for the first time report that IL-22 is expressed by IL-17expressing TH cells and is regulated in the same manner as IL-17. Since IL-22 and IL-17 do not have any effect in regulation of TH differentiation, they may jointly regulate tissue inflammation. 


\section{Materials and Methods}

\section{T-cell differentiation}

OVA-specific CD4T cells isolated from OT-II mice using antiCD4-microbeads by AutoMACS (Miltenyi Biotec) were cultured with irradiated splenic APC from $\mathrm{C} 57 \mathrm{BL} / 6$ mice prepared as described previously [4] in the presence of $\mathrm{OVA}_{323-339}$ peptide. For TH1 differentiation, anti-IL-4 (11B11, $10 \mu \mathrm{g} / \mathrm{ml})$ and IL-12 $(2 \mathrm{ng} / \mathrm{ml})$ were added. For TH2 differentiation, anti-IFN $\gamma(\mathrm{XMG} 1.2,10 \mu \mathrm{g} / \mathrm{ml})$ and IL-4 (10 ng/ml) were added. For THi differentiation, anti-IL-4/antiIFN $\gamma$ Abs, IL-6 (10 ng/ml), TGF $\beta$ (5 ng/ml), IL-23 (5 ng/ml) were used. To examine the effect of IL-22 during Th 17 differentiation, recombinant human IL-22 $(100 \mathrm{ng} / \mathrm{ml})$ or anti-mIL-22 polyclonal Abs ( $5 \mu \mathrm{g} / \mathrm{ml}, \mathrm{R} \& \mathrm{D}$ systems) were added into the cell culture.

\section{Real-time RT-PCR analysis}

$\mathrm{T}$ cells differentiated for 5 days were restimulated with platebound anti-CD3 $\mathrm{Ab}(2 \mathrm{C} 11,5 \mu \mathrm{g} / \mathrm{ml})$ for $5 \mathrm{~h}$. Total RNA was extracted from these cells with TRIzol reagent (Invitrogen) and the cDNA was generated with an oligo(dT) primer followed by analysis using iCycler PCR with the iQTM SYBR ${ }^{\circledR}$ Green Supermix (Bio-Rad) based on expression of $\beta$-actin. The following primer pairs were used: $\beta$-actin: F-GAC GGC CAG GTC ATC ACT ATT G and R-AGG AAG GCT GGAAAA GAG CC; IFN $\gamma$ : F-GAT GCA TTC ATG AGT ATT GCC AAG T and R-GTG GAC CAC TCG GAT GAG CTC; IL-4: F-AGA TCA CGG CAT TTT GAACG and R-TTT GGC ACA TCC ATC TCC G; IL-17: F-CTG GAG GAT AAC ACT GTG AGA GT and R-TGC TGA ATG GCG ACG GAG TTC; IL-22: F-CAT GCA GGA GGT GGT ACC TT and R-CAG ACG CAA GCA TTT CTC AG.

\section{ELISA}

T cells $\left(1 \times 10^{6} /\right.$ well $)$, after differentiation for 5 days as described above, were restimulated with plate-bound anti-CD3 Ab $(5 \mu \mathrm{g} / \mathrm{ml})$ in 24-well plates. Twenty-four hours later, culture supernatant was harvested, and the concentration of IFN- $\gamma$, IL-4, IL-17 (BD Bioscience) and IL-22 (Antigenix) was measured by ELISA [4], following the manufacturer's direction.

\section{Intracellular cytokine staining}

$\mathrm{T}$ cells, after differentiation for 5 days, were restimulated with PMA $(50 \mathrm{ng} / \mathrm{ml})$ and ionomycin $(500 \mathrm{ng} / \mathrm{ml})$ in the presence of GolgiPlug (BD Bioscience) for $5 \mathrm{~h}$. These cells were stained with FITC-conjugated anti-V $\alpha 2$ Abs then permeabilized as per the manufacturer's instruction. Cells were additionally stained with PE-conjugated anti-IL-17 and APC-conjugated anti-IFN $\gamma$ Abs before FACSCalibur analysis.

\section{Results}

\section{Expression of IL-22 in IL-17-producing TH cells}

In order to identify genes that are selectively expressed in different subsets of $\mathrm{TH}$ cells, we recently compared gene expression profiles of in vitro differentiated TH1, TH2 and THi cells. In addition to IL-17 that was highly upregulated by THi cells, we found that IL-22 expression was also selectively upregulated in these cells $(>8 \times$ than TH1 cells and $>16 \times$ than TH2). This result was further confirmed by real-time RT-PCR analysis. CD4+ T cells isolated from OT-II TcR transgenic mice were activated by Ova peptide and splenic APC and differentiated into TH1 (in the presence of IL-12 and anti-IL-4), TH2 (IL-4 and anti-IFN $\gamma$ ) or THi (IL-6, TGF $\beta$, IL-23, anti-IL-4 and anti-IFN $\gamma$ ) cells. While IFN $\gamma$ gene was highly expressed in TH1 cells and IL-4 was highly expressed in TH2 cells, expression of IL-17 and IL-22 was restricted to THi cells (Figure 1A).

To further characterize the expression of IL-22 in different $\mathrm{TH}$ cells, we examined IL-22 protein levels in their supernatants by ELISA following anti-CD3 restimulation. Consistent with the results of real-time RT-PCR analysis, the production of IFN $\gamma$, IL-4 and IL-17 was highly restricted to TH1, TH2 and THi, respectively (Figure 1B). The production of IL-22 by TH1 was higher than by TH2; however, this cytokine was produced at greatly increased levels in THi cells $(>2.5 \times$ than TH1 cells and $>10 \times$ than TH2; Figure 1B). Taken together, these data indicate that IL-22 may be another cytokine uniquely expressed in the THi subset.

\section{Coordinated regulation of $I L-17$ and $I L-22$ expression during TH differentiation}

Recent studies have indicated IL-23 or TGF $\beta$ and IL-6 as cytokines regulating THi cell differentiation $[3,7]$. To substantiate our above findings, we further differentiate OT-II cells under various conditions and assessed the regulation of IL-22 expression in comparison to that of IL-17, IFN $\gamma$ or IL-4. When OT-II cells were differentiated in the presence of IL- 6 or TGF $\beta$, there was little IL-22 or IL-17 mRNA expression (Figure 2A). On the other hand, combination of these two cytokines led to greatly increased levels of IL-22 and IL-17 expression and downregulation of IFN $\gamma$ and IL-4 expression (Figure 2A). IL-23 also moderately enhanced IL-22 and IL-17 expression. Combination of IL-6, TGF $\beta$ and IL-23, while inhibiting TH1 and TH2 differentiation by blocking IFN $\gamma$ and IL-4, led to most optimal mRNA expression of both IL-22 and IL-17 (Figure 2A).

When we analyzed the protein expression of IFN $\gamma$, IL-4 and IL-17 from T cells differentiated under the same condition, we observed similar results (Figure 2B). The addition of IL-6 appeared to induce IL-22 substantially, although we did not see any significant increase in its mRNA level (Figure 2B). Moreover, combination of IL-6 and TGF $\beta$ led to a further increase in IL-22 production, although TGF $\beta$ alone suppressed the IL-22 production. Further inclusion of IL-23 plus neutralizing Abs against IFN $\gamma$ and IL-4 led to most optimal production of IL-22 cytokine, consistent with real-time RT-PCR analysis (Figure 2B). All these data indicate coordinated regulation of IL-17 and IL-22 expression, which further supports IL-22 as a novel cytokine 
A
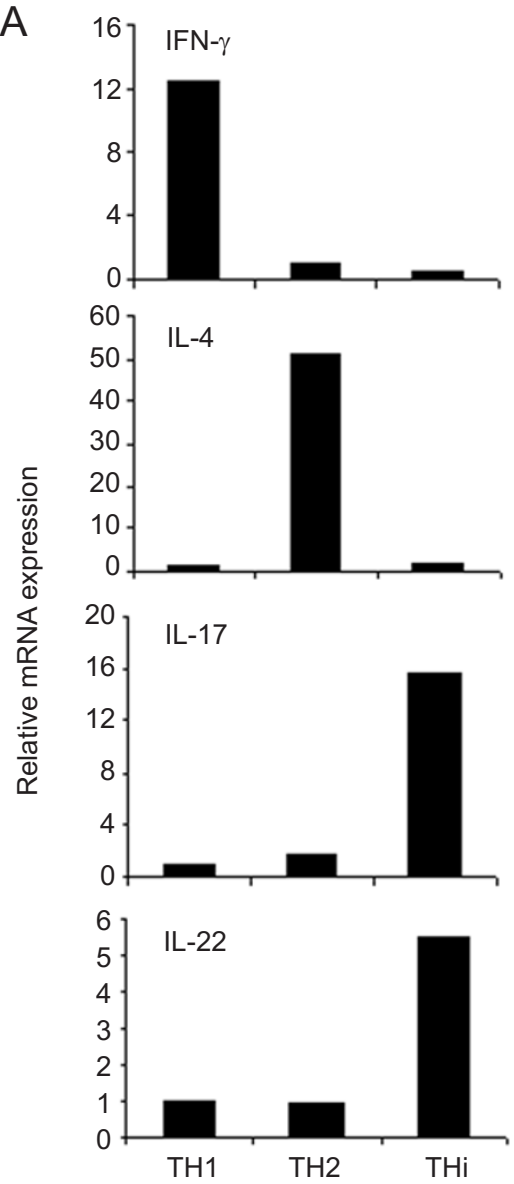

B
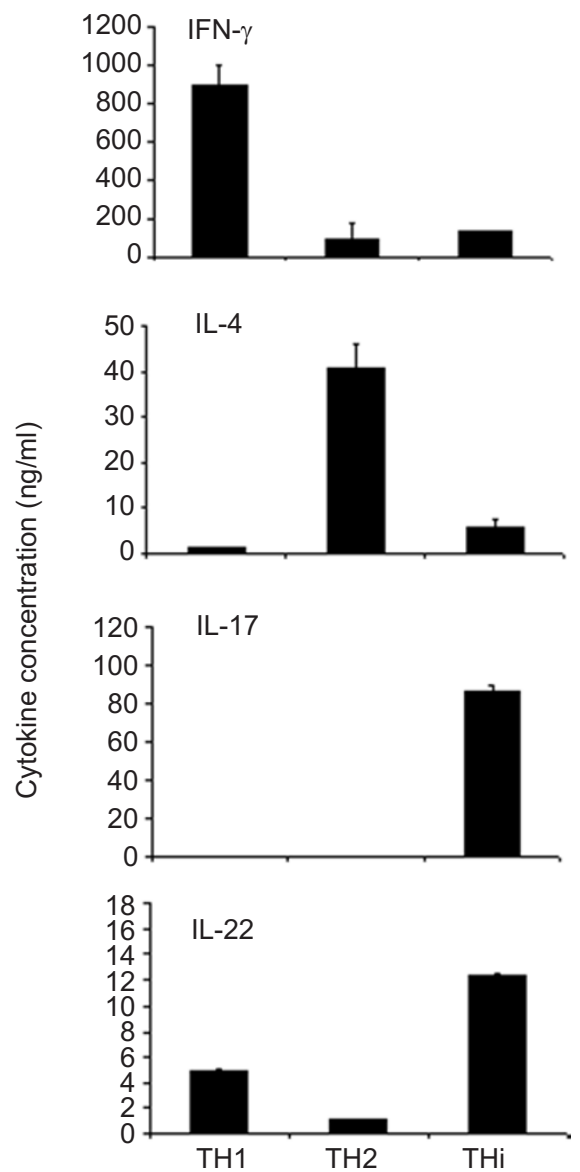

Figure 1 Preferential expression of IL-22 in THi cells. (A, B) Naïve CD4+ T cells isolated from OT-II mice were differentiated into TH1, TH2 or THi cells. (A) Five days later, cells were restimulated with plate-bound anti-CD3 Ab and analyzed for cytokine mRNA levels by real-time RT-PCR. Results are normalized to $\beta$-actin. Data are representative of two independent experiments. (B) Five days later, $\mathrm{T}$ cells were restimulated with plate-bound anti-CD3 Ab for $24 \mathrm{~h}$ and the expression of IFN- $\gamma$, IL-4, IL-17 and IL-22 in the supernatant was measured by ELISA. Bars are mean \pm SD.

expressed in THi cells.

\section{IL-22 does not regulate TH differentiation}

While IFN $\gamma$ regulates TH1 differentiation and IL-4 mediates TH2 differentiation, it is unclear whether there is a cytokine produced by THi cells to regulate their own differentiation. We thus tested the ability of IL-22 in THi differentiation. Addition of exogenous IL-22 to the above OT-II differentiation system did not augment THi differentiation under suboptimal or optimal conditions; IL-17 and IFN $\gamma$ expression was not affected by an intracellular cytokine staining assay (Figure 3). We also utilized an antibody against IL-22 in the culture to block the effect of IL-22 produced by T cells. This antibody did not affect THi differentiation either (Figure 3). Thus, IL-22 does not regulate T-cell differentiation.

\section{Discussion}

Upon activation, naïve TH cells differentiation into different subsets of effector $\mathrm{T}$ cells that are specialized in their cytokine expression and immune function. A novel lineage of TH cells that produce IL-17 was recently identified and strongly implicated in host responses to infection and in autoimmune diseases. Here, we report an additional cytokine product expressed in these cells - IL-22.

In a gene-chip analysis of TH1, TH2 and THi cells, we found IL-22 mRNA was significantly upregulated in THi cells compared with the other two subsets. The idea that IL-22 is a THi cytokine was further supported by a realtime RT-PCR and ELISA analysis of IL-22 expression in these subsets (Figure 1). Furthermore, in analysis of TH differentiation conditions that result in IL-22 expression, 
A

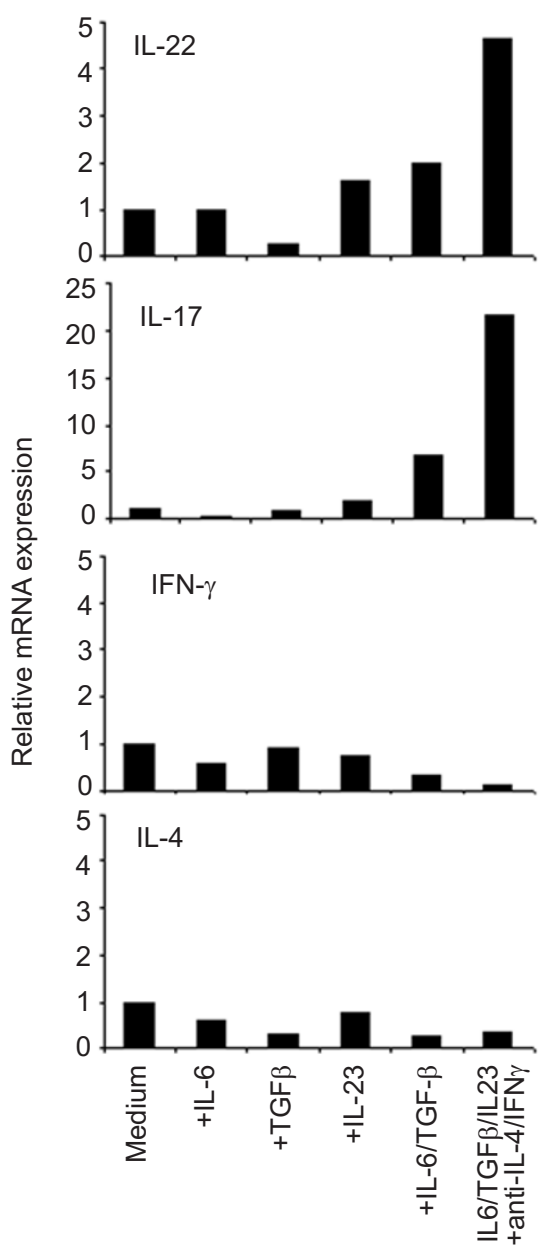

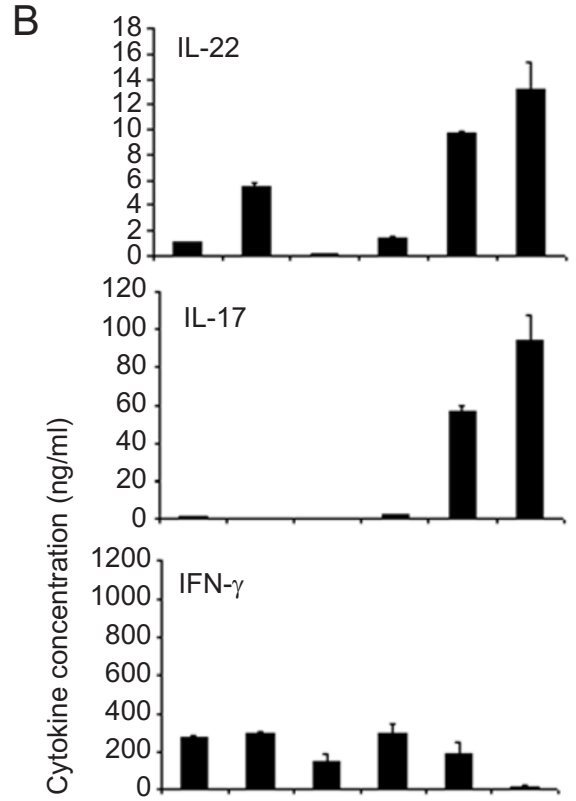

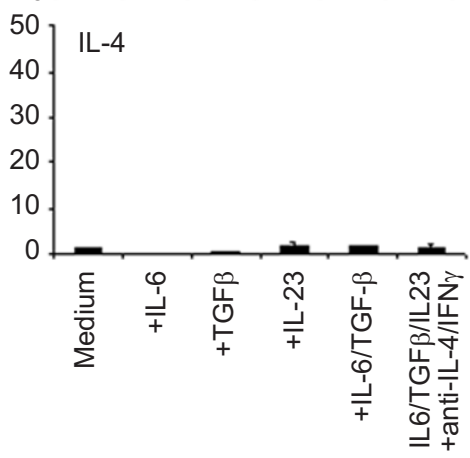

Figure 2 Coordinated regulation of IL-22 and IL-17 in TH differentiation. (A, B) CD4T cells isolated from OT-II mice were activated in the presence of indicated cytokines or cytokine blockers. (A) Five days later, cells were restimulated with plate-bound anti-CD3 Ab and analyzed for cytokine mRNA levels by real-time RT-PCR. Results are normalized to $\beta$-actin. Expression level in medium alone was set to 1. (B) Five days later, T cells were restimulated with plate-bound anti-CD3 $\mathrm{Ab}$ for $24 \mathrm{~h}$ and the concentration of IFN- $\gamma$, IL-4, IL-17 and IL-22 in the supernatant was measured by ELISA. Bars are mean $\pm \mathrm{SD}$.

we found that the mRNA and protein expression of IL-17 and IL-22 were coordinately regulated (Figure 2). All these data indicate that IL-22 is expressed by IL-17-producing THi cells. However, at this stage, we do not know whether these two cytokines are expressed by the same or different cells at a single-cell level. The intracellular protein analysis of these cytokines is needed in the future. In addition, their relationship with IL-17F, another cytokine expressed in the THi cells $[3,7]$ needs to be addressed.

In our TH cell differentiation experiments, we did not find any significant effect by exogenous IL-22 or inhibiting IL-22 on subsequent cytokine expression by the effector cells (Figure 3). This further supports the previous conclusion that immune cells may not be the targets of IL-22.
Similarly, IL-17 also mainly functions to promote tissue inflammation in vitro and in vivo [4]. With this in mind, IL-17 and IL-22 may have synergistic function on their common target tissues or cells. IL-17 strongly induced proinflammatory cytokine expression in mouse fibroblasts [4]. However, we did not observe any synergistic effect by IL-22 in these cells (data not shown). Epithelial cells may be a common target cell type of both cytokines $[4,10]$. It has been reported that IL-22 in plasma of psoriatic patients is higher than that of healthy individuals [11]. This cytokine induces keratinocytes to produce anti-microbial peptides and S100 proteins, chemokines that recruit neutrophils. On the other hand, the association of microbial infection and chronic inflammation has been suggested in autoim- 


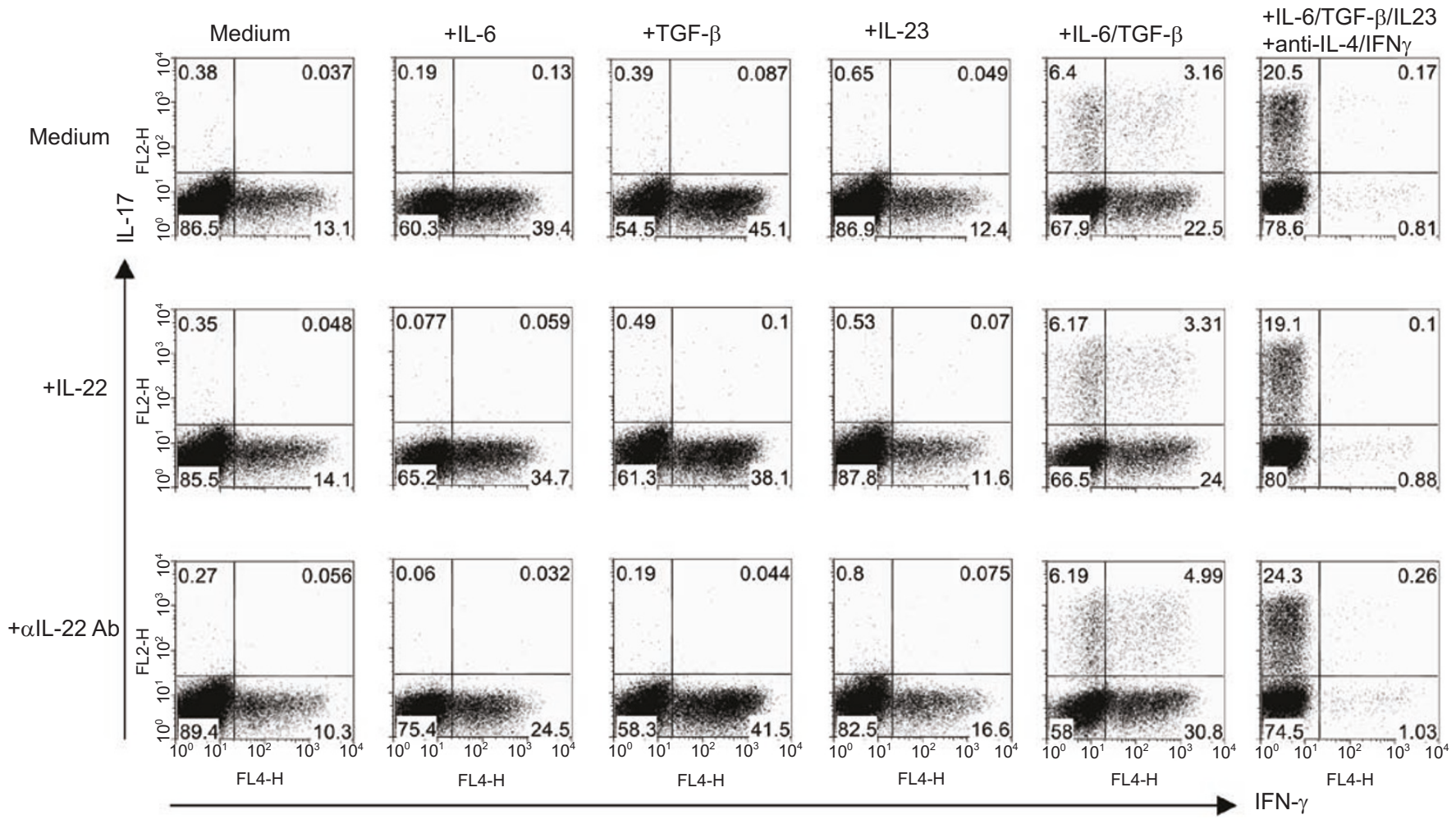

Figure 3 IL-22 does not regulate the generation of THi in vitro. CD4+ T cells isolated from OT-II mice were activated in the presence of indicated cytokines or cytokine blockers. IL-22 or anti-IL-22 polyclonal Abs were added into indicated samples. Five days later, cells were restimulated with PMA and ionomycin for $5 \mathrm{~h}$ and analyzed for intracellular IL-17 and IFN $\gamma$ expression on gated CD4+ V 2 2+ OT-II cells.

mune disease models including psoriasis [12]. Based on our findings in the present study, we propose that these two cytokines secreted from THi cells synergize to promote autoimmunity and tissue inflammation such as psoriasis. IL-22 and IL-17 may be crucial mediators of this inflammatory disorder in different stages of pathogenesis and may have effects on different target cells.

IL-17-expressing TH cells have recently emerged as an attractive target for immunotherapy against autoimmune diseases. While IL-17 itself has potent inflammatory function, our current study has added another layer of complexity on these cells. One may need to consider the effect of IL-22 in THi-mediated diseases. Understanding the function and regulation of IL-22 would certainly be beneficial in the future in treatment of inflammatory diseases such as dermatitis and psoriasis in which IL-22 was found overexpressed.

\section{Acknowledgment}

We thank Bruz Marzolf at the Microarray Facility at Institute for Systems Biology for technical assistance and the entire Dong Lab for discussion and help. This work was in part supported by National Institutes of Health (to CD). SHC receives a post-doctoral fellowship from the Arthritis foundation and $\mathrm{CD}$ is a Cancer Research Institute Investigator and an MD Anderson Cancer Center Trust Fellow.

\section{References}

1 Glimcher LH, Murphy KM. Lineage commitment in the immune system: the T helper lymphocyte grows up. Genes Dev 2000; 14:1693-1711.

2 Dong C, Flavell RA. Cell fate decision: T-helper 1 and 2 subsets in immune responses. Arthritis Res 2000; 2:179-188.

3 Dong C. Diversification of T-helper-cell lineages: finding the family root of IL-17-producing cells. Nat Rev Immunol 2006; 6:329-334.

4 Park H, Li Z, Yang XO, et al. A distinct lineage of CD4T cells regulates tissue inflammation by producing interleukin 17 . Nat Immunol 2005; 6:1133-1141.

5 Dong C, Nurieva RI. Regulation of immune and autoimmune responses by ICOS. J Autoimmun 2003; 21:255-260.

6 Langrish CL, Chen Y, Blumenschein WM, et al. IL-23 drives a pathogenic $\mathrm{T}$ cell population that induces autoimmune inflammation. J Exp Med 2005; 201:233-240. 
7 Weaver CT, Harrington LE, Mangan PR, Gavrieli M, Murphy KM. Th17: an effector CD4T cell lineage with regulatory T cell ties. Immunity 2006; 24:677-688.

8 Xie MH, Aggarwal S, Ho WH, et al. Interleukin (IL)-22, a novel human cytokine that signals through the interferon receptor-related proteins CRF2-4 and IL-22R. J Biol Chem 2000; 275:31335-31339.

9 Pestka S, Krause CD, Sarkar D, Walter MR, Shi Y, Fisher PB. Interleukin-10 and related cytokines and receptors. Annu Rev Immunol 2004; 22:929-979.
10 Wolk K, Kunz S, Witte E, Friedrich M, Asadullah K, Sabat R. IL-22 increases the innate immunity of tissues. Immunity 2004; 21:241-254.

11 Wolk K, Witte E, Wallace E, et al. IL-22 regulates the expression of genes responsible for antimicrobial defense, cellular differentiation, and mobility in keratinocytes: a potential role in psoriasis. Eur J Immunol 2006; 36:1309-1323.

12 Karin M, Lawrence T, Nizet V. Innate immunity gone awry: linking microbial infections to chronic inflammation and cancer. Cell 2006; 124:823-835. 